\title{
Enfoque familiar e comunitário da Atenção Primária à Saúde a pessoas com Hipertensão Arterial
}

\author{
Family and community approach to Primary Health Care for people \\ with Hypertension
}

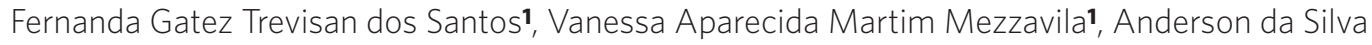
Rêgo', Maria Aparecida Salci', Cremilde Aparecida Trindade Radovanovic ${ }^{\mathbf{1}}$

DOI: 10.1590/0103-1104201912116

1 Universidade Estadual de Maringá (UEM) Departamento de Enfermagem, Programa de Pós-Graduação em Enfermagem (PSE) Maringá (PR), Brasil. anderson.dsre@hotmail.com
RESUMO A Hipertensão Arterial é uma morbidade crônica de alta prevalência no mundo. Nesse prisma, é importante conhecer o impacto da assistência prestada pelos profissionais, no contexto da Atenção Primária à Saúde, para compreensão e formulação de novas estratégias de atendimento. Este estudo objetivou analisar o enfoque familiar e comunitário da Atenção Primária à Saúde a pessoas com Hipertensão Arterial. Trata-se de um estudo transversal, realizado com 417 pessoas em tratamento da Hipertensão Arterial de 34 Unidades Básicas de Saúde do município de Maringá, localizado no noroeste do estado do Paraná, Brasil. Os dados foram coletados no primeiro semestre de 2016, utilizando instrumento adaptado e validado, que aborda atributos da Atenção Primária à Saúde, sendo utilizado, neste estudo, o atributo enfoque familiar e comunitário. Para tratamento das variáveis, foi utilizada análise inferencial. Foi possível observar que os atributos referentes ao envolvimento familiar no tratamento, incentivo à participação social na comunidade e realização de educação em saúde foram os quesitos com pior avaliação, sendo representados estatisticamente por pessoas com controle pressórico inadequado. Conclui-se que a atenção quanto ao enfoque familiar e comunitário possui fragilidades e que necessitam ser reavaliadas, integrando a família e a comunidade no tratamento de pessoas com Hipertensão Arterial.

PALAVRAS-CHAVE Hipertensão. Atenção Primária à Saúde. Pesquisa sobre serviços de saúde. Família. Integração comunitária.

ABSTRACT Hypertension is a chronic, high prevalence morbidity in the world. In this aspect, it is important to know the impact of care provided by professionals, in the context of Primary Health Care, to understand and formulate new care strategies. This study aimed to analyze the family and community approach in Primary Health Care for people with arterial Hypertension. This is a cross-sectional study carried out with 417 people undergoing treatment for arterial Hypertension in 34 Basic Health Units of the city of Maringá, located in the northwestern part of the state of Paraná, Brazil. The data were collected in the first half of 2016, using an adapted and validated instrument, which addresses the attributes of Primary Health Care, using the family and community approach in this study. For the treatment of variables, descriptive and inferential statistical analysis was used. It was possible to observe that the attributes related to family involvement 
in the treatment, incentive to social participation in the community, and health education were the items with worse evaluation, being represented statistically by people with inadequate blood pressure control. It is concluded that the care to the family and community focus has weaknesses and needs to be re-evaluated, integrating the family and the community in the treatment of people with arterial Hypertension.

KEYWORDS Hypertension. Primary Health Care. Health services research. Family. community integration.

\section{Introdução}

A Hipertensão Arterial (HA) é uma doença crônica, considerada um problema de saúde pública mundial devido a sua carga incapacitante e às complicações oriundas de sua cronicidade. A prevalência da HA é de aproximadamente $31 \%$, responsável por $63 \%$ de um total de 38 milhões de óbitos mundiais de adultos ${ }^{\mathbf{1} 2}$. No Brasil, até o ano de 2017, a prevalência da HA era de aproximadamente $24,3 \%$ em indivíduos adultos, sendo as mulheres $(26,4 \%)$ com prevalência maior em relação aos homens $(21,7 \%)^{3}$.

O tratamento da HA é frequentemente auxiliado na integração de medicamentos para o controle pressórico, com alteração nos hábitos de vida, com promoção e adoção de práticas de exercício físico e mudança nos hábitos nutricionais e alimentar. Com o tempo, essas mudanças acarretam alterações emocionais que a pessoa com HA enfrenta para adaptação ao novo estilo de vida e convivência com a morbidade, afetando sua vida social, cultural, econômica, bem como atingindo os seus familiares e amigos, o que pode comprometer a adesão ao tratamento $\mathbf{1 , 4 , 5}$.

No que tange à acessibilidade ao tratamento, a Atenção Primária à Saúde (APS) consolida vários atributos para o serviço de saúde, articulando uma assistência programática e complementar, tornando-se a principal porta de entrada do sistema, completando e organizando a assistência, sendo o acesso, a acessibilidade, a adesão/o vínculo, o elenco de serviços, a coordenação do cuidado, o enfoque familiar e comunitário seus principais atributos ${ }^{6,7}$. Cabe à APS ser o suporte primordial e básico à população, ampliando os serviços em saúde, com abordagem das morbidades, das premências e dos processos que compõem a saúde, que caracterizam o processo coletivo e/ou unitário, tornando-se ponto de comunicação para os demais serviços que compõem a Rede de Atenção à Saúde, caracterizando a gestão da atenção ${ }^{6,7}$.

No âmbito do enfoque familiar, o papel dos profissionais de saúde em relação à assistência prestada à pessoa com HA pode ser evidenciado por meio das práticas assistenciais, inserindo a família como unidade de cuidado, como também em descrever os caminhos percorridos na busca por tratamento. Assim, as mudanças produzidas pelo diagnóstico de uma morbidade crônica, a adesão ao tratamento, as características sociais e culturais e os fatores emocionais acarretam alterações na dinâmica familiar que necessitam ser investigadas ${ }^{\mathbf{8}, 9}$.

No que concerne à participação comunitária para tomada de decisões sobre as ações de saúde, esse atributo oferece parâmetros que muitas vezes não são compreendidos pelos 
profissionais responsáveis pela atenção à determinada comunidade. No entanto, as pessoas com HA possuem fatores que justificam a não adoção ao tratamento, como infraestrutura inadequada, violência urbana e condições de saneamento básico. Não obstante, a população ainda tende a procurar atendimento médico apenas com a presença da doença; portanto, a prevenção necessita ser disseminada para que se promova um ambiente saudável para a comunidade 9,10 .

Nesse aspecto, a avaliação dos serviços ofertados para as pessoas com HA, com destaque ao enfoque familiar e comunitário, é relevante para os serviços e programas em saúde fornecidos pela APS. Apesar de ser pouco explorada, a avaliação da satisfação dos usuários dos serviços de saúde fornece informações que podem contribuir para a formulação de novas estratégias e manejo das ações de saúde adequadas às características desses grupos 7,9-12. Diante disso, o objetivo deste estudo é analisar o enfoque familiar e comunitário da APS a pessoas com HA.

\section{Material e métodos}

O estudo faz parte de uma pesquisa maior, intitulada 'Avaliação da satisfação de pessoas com Hipertensão Arterial com os serviços da Atenção Primária à Saúde'. O projeto do qual este estudo faz parte seguiu as consonâncias da Resolução $n^{0} 466 / 2012^{13}$, sendo aprovado pelo Comitê Permanente de Ética em Pesquisa com Seres Humanos, da Universidade Estadual de Maringá (UEM) sob o parecer de número 1.407.687/2016. O Termo de Consentimento Livre e Esclarecido (TCLE) foi disponibilizado em duas vias de igual teor, que foram lidas e assinadas por todos os participantes.

Trata-se de um estudo de abordagem transversal, realizado no município de Maringá, interior do estado do Paraná, Brasil. O município possui população estimada em 406.693 habitantes até o ano de 2016, com 34 Unidades Básicas de Saúde (UBS) atendendo de forma descentralizada e com auxílio de 74 equipes da
Estratégia Saúde da Família (ESF), perfazendo a cobertura populacional de $68 \%{ }^{\mathbf{1 4}}$.

Para a definição do cálculo amostral, baseou-se no número de 27.741 indivíduos que estavam em tratamento da HA e que se encontravam cadastrados no programa Sishiperdia. Utilizou-se, como parâmetros, a estimativa de erro de $5 \%$, com $95 \%$ de intervalo de confiança e acrescida, para possíveis perdas, uma taxa de $15 \%$. Após o cálculo, foi realizada a amostra estratificada dos usuários cadastrados em cada UBS incluída na pesquisa. A Secretaria Municipal de Saúde disponibilizou a lista, com nome e código do cadastro no sistema interno da rede de atenção à saúde, que serviu de base para a realização de um sorteio aleatório conforme o número de pessoas atendidas em cada UBS. Com as perdas e recusas, a amostra final foi composta por 417 pessoas.

Os pacientes inclusos no estudo tinham 18 anos ou mais, de ambos os sexos, com visitas regulares ao atendimento fornecido pelas UBS, cadastrados no Sishiperdia há um ano antes da coleta de dados, para que houvesse o tempo de no mínimo 12 meses para responderem às perguntas relativas a este estudo. Os critérios de exclusão foram pacientes sem condições clínicas (dificuldades de verbalização) para participar do estudo, aqueles que não realizaram consultas anteriores até o momento da coleta de dados e gestantes.

Antes do início da coleta de dados, os gestores das UBS e enfermeiros responsáveis pelas reuniões do Sishiperdia foram abordados pelos pesquisadores, em que foram apresentados os objetivos do estudo e sua participação durante o procedimento. As reuniões aconteceram em horário fixo, que variou das $8 \mathrm{~h}$ às $17 \mathrm{~h}$, de acordo com a organização das 74 equipes da ESF inclusas no estudo. Posteriormente, todos os participantes foram esclarecidos sobre informações da pesquisa; e a coleta de dados ocorreu nas dependências das UBS, em lugares reservados e livre de intercorrências.

Participaram da coleta de dados dois enfermeiros estudantes de doutorado, vinculados a um programa de pós-graduação de uma 
universidade do município, e quatro alunos de graduação em enfermagem, vinculados a um grupo de pesquisa da mesma instituição. Para todos os participantes da coleta de dados, foram dadas orientações sobre os procedimentos a serem realizados quanto a preenchimento dos instrumentos e abordagem aos partícipes. Também foi realizado teste piloto para verificar a necessidade de mudança com os instrumentos realizados, não sendo necessário alteração quanto ao seu conteúdo.

Para a coleta de dados, foram utilizados dois instrumentos e verificada a pressão arterial de todos os participantes. O primeiro instrumento foi utilizado para estimar o poder de compra dos entrevistados e de sua família, agrupados em seis classes: A, B1, B2, C1, C2 e DE ${ }^{15}$.
O segundo instrumento avaliou a satisfação individual do usuário do serviço de saúde diagnosticados com HA quanto aos profissionais colaboradores da UBS e aos serviços prestados pela APS. Esse instrumento foi elaborado e validado em duas cidades do nordeste brasileiro e teve como apoio o Primary Care Assessment Tool (PCATool), estudo desenvolvido pela Johns Hopkins Primary Care Policy Center, com os pressupostos de Starfield 7 .

Os domínios do instrumento são divididos em acesso ao diagnóstico, acessibilidade ao tratamento, adesão/vínculo, elenco de serviços, coordenação, enfoque familiar e orientação comunitária ${ }^{16}$. Para este estudo, utilizaram-se os indicadores referentes ao enfoque na família e orientação comunitária (quadro 1).

Quadro 1. Indicadores de enfoque familiar e orientação comunitária a pessoas com Hipertensão Arterial na Atenção Primária à Saúde. Maringá, Paraná, Brasil, 2016

\begin{tabular}{|c|c|}
\hline Indicadores & Dimensões \\
\hline \multicolumn{2}{|c|}{ Enfoque familiar } \\
\hline EF1 & $\begin{array}{l}\text { Os profissionais da unidade de saúde procuram conhecer as pessoas que moram com } \\
\text { o(a) Sr.(a)? }\end{array}$ \\
\hline EF2 & $\begin{array}{l}\text { Os profissionais da unidade de saúde conversam com as pessoas que moram com o(a) } \\
\text { Sr.(a) sobre a HA, estilo de vida, o seu tratamento e outros problemas de saúde? }\end{array}$ \\
\hline EF3 & $\begin{array}{l}\text { Os profissionais de saúde conversam sobre a importância do envolvimento da sua } \\
\text { família no seu tratamento? }\end{array}$ \\
\hline \multicolumn{2}{|c|}{ Orientação comunitária } \\
\hline $\mathrm{OC1}$ & $\begin{array}{l}\text { Os profissionais da unidade de saúde conversam sobre a importância da sua participa- } \\
\text { ção e da participação da sua família em instituições da comunidade (igrejas, associação } \\
\text { de bairro etc.) como apoio para resolver seus problemas de saúde? }\end{array}$ \\
\hline OC2 & $\begin{array}{l}\text { Os profissionais da unidade de saúde conversam sobre a influência da família/amigos/ } \\
\text { colegas no seu tratamento? }\end{array}$ \\
\hline OC3 & $\begin{array}{l}\text { Com que frequência os serviccos de saúde desenvolvem ações sobre HA com as igrejas, } \\
\text { associações de bairro, escolas? }\end{array}$ \\
\hline
\end{tabular}

Fonte: Paes, 201416

As questões de cada dimensão são constituídas por proporcionalidades, chamada escala de Likert, atribuindo-se valores entre um e cinco, respectivamente, para as respostas: 1) 'nunca'; 2) 'quase nunca'; 3) 'às vezes'; 4) 'quase sempre' e 5) 'sempre’16. A classificação da avaliação foi realizada de acordo com os seguintes pontos de corte: satisfatório $(\geq 4)$, regular $(<4$ e $\geq 3)$ e insatisfatório $(<3)^{8}$.

A pressão arterial foi aferida durante os procedimentos de coleta de dados, seguindo as diretrizes e orientações da VII Diretriz 
Brasileira de Hipertensão Arterial ${ }^{17}$. Os valores coletados foram categorizados em adequado quando a pressão arterial sistólica (PAS) $\leq 140$ mmHg e pressão arterial diastólica (PAD) $\leq$ $90 \mathrm{mmHg}$ e inadequado quando $\mathrm{PAS} \geq 140$ $\mathrm{mmHg}$ e $\mathrm{PAD} \geq 90 \mathrm{mmHg}$.

Os dados foram duplamente digitados em planilha eletrônica Microsoft Excel ${ }^{\circledR}$ 2016; e realizada análise estatísticas com o auxílio do software SPSS, versão 19.0. Realizou-se a identificação de normalidade dos dados pelo teste de Kolmogorov-Smirnov, com correção de Lilliefors. Foi realizada análise descritiva dos resultados referente à situação sociodemográfica e categorização dos valores de pressão arterial.

Para a análise de variância, foi utilizado o teste de Kruskall-Wallis para obter a média dos escores dos domínios, medida de dispersão e para indicar se houve diferença nas médias em relação aos grupos de controle pressórico adequado e inadequado. Para melhor visualização dos dados, foi realizada a apresentação dos valores medianos em diagramas de caixas, por meio de quartis, divididos entre a classificação do controle pressórico, sem suposição hipotética.

\section{Resultados}

Foram entrevistadas 417 pessoas em tratamento da HA. Destas, a maioria era idosa $(31,9 \%)$, do sexo feminino $(67,9 \%)$, da cor branca $(62,4 \%)$. Quanto às questões sociais, grande parte tinha até o ensino fundamental $(61,2 \%)$, era casada $(58,3 \%)$, classificadas em extrato C2 (24,0\%); e a maioria era aposentada/ pensionista $(55,2 \%)$ (tabela 1 ). Não houve diferença nos grupos de controle pressórico adequado e inadequado, estando os valores de porcentagens alusivos à caracterização total da população, como pode ser observado na tabela 1.

Tabela 1. Dados sociodemográficos e econômico de pessoas com Hipertensão Arterial acompanhada pela Atenção Primária à Saúde. Maringá, Paraná, Brasil, 2016

\begin{tabular}{|c|c|c|c|c|c|c|}
\hline & \multirow[b]{3}{*}{$\mathrm{N}$} & \multirow{3}{*}{$\begin{array}{r}\text { Total } \\
\% \\
\end{array}$} & \multicolumn{4}{|c|}{ Controle Pressórico } \\
\hline & & & \multicolumn{2}{|c|}{ Adequado $(n=224)$} & \multicolumn{2}{|c|}{ Inadequado $(n=193)$} \\
\hline & & & $\mathbf{N}$ & $\%$ & $\mathbf{N}$ & $\%$ \\
\hline \multicolumn{7}{|l|}{ Idade } \\
\hline $20-29$ & 15 & 3,6 & 9 & 4 & 6 & 3,1 \\
\hline $30-39$ & 25 & 6 & 11 & 4,9 & 14 & 7,3 \\
\hline $40-49$ & 37 & 8,9 & 24 & 10,7 & 13 & 6,7 \\
\hline $50-59$ & 80 & 19,2 & 51 & 22,8 & 29 & 15,0 \\
\hline $60-69$ & 127 & 30,5 & 64 & 28,6 & 63 & 32,6 \\
\hline$>70$ & 133 & 31,9 & 65 & 29,0 & 68 & 35,2 \\
\hline \multicolumn{7}{|l|}{ Sexo } \\
\hline Masculino & 134 & 32,1 & 74 & 33,0 & 60,0 & 31,1 \\
\hline Feminino & 283 & 67,9 & 150 & 67,0 & 133,0 & 68,9 \\
\hline \multicolumn{7}{|l|}{ Raça/Cor } \\
\hline Branca & 260 & 62,4 & 138 & 61,6 & 122 & 63,2 \\
\hline Preta & 65 & 15,6 & 37 & 16,5 & 28 & 14,5 \\
\hline Parda & 92 & 22,1 & 49 & 21,9 & 43 & 22,6 \\
\hline
\end{tabular}


Tabela 1. (cont.)

\begin{tabular}{|c|c|c|c|c|c|c|}
\hline \multicolumn{7}{|l|}{ Escolaridade } \\
\hline Não sabe ler/escrever & 32 & 7,7 & 20 & 8,9 & 12 & 6,2 \\
\hline Ensino Fundamental & 255 & 61,2 & 131 & 58,5 & 124 & 64,2 \\
\hline Ensino Médio & 107 & 25,7 & 61 & 27,2 & 46 & 23,8 \\
\hline Ensino Superior & 23 & 5,5 & 12 & 5,4 & 11 & 5,7 \\
\hline \multicolumn{7}{|l|}{ Situação Conjugal } \\
\hline Sem Companheiro(a) & 174 & 41,7 & 97 & 43,3 & 77 & 39,9 \\
\hline Com Companheiro(a) & 243 & 58,3 & 127 & 57,5 & 116 & 60,1 \\
\hline \multicolumn{7}{|l|}{ Classificação Econômica - Abep* } \\
\hline A & 19 & 4,6 & 10 & 4,5 & 9 & 4,7 \\
\hline B1 & 33 & 7,9 & 18 & 8 & 15 & 7,8 \\
\hline B2 & 96 & 23,0 & 47 & 21 & 49 & 25,4 \\
\hline $\mathrm{C} 1$ & 83 & 19,9 & 41 & 18,3 & 42 & 21,8 \\
\hline $\mathrm{C} 2$ & 100 & 24,0 & 57 & 25,4 & 43 & 22,3 \\
\hline DE & 86 & 20,6 & 51 & 22,8 & 35 & 18,0 \\
\hline \multicolumn{7}{|l|}{ Ocupação Atual } \\
\hline Empregado & 96 & 23 & 58 & 25,9 & 38 & 19,7 \\
\hline Desempregado & 91 & 21,8 & 56 & 25 & 35 & 18,1 \\
\hline Aposentado(a)/Pensionista & 230 & 55,2 & 110 & 49,1 & 120 & 62,2 \\
\hline
\end{tabular}

Fonte: Elaboração própria.

*Abep - Associação Brasileira de Empresas de Pesquisa.

$\mathrm{Na}$ análise do atributo enfoque familiar, a questão relacionada ao envolvimento da família no tratamento apresentou menor média (EF3) $(2,98 \pm 1,45)$, seguida do interesse dos profissionais de saúde em conhecer as pessoas que moram na mesma residência do entrevistado
(EF1) $(3,04 \pm 1,45)$. Na análise de variância, os resultados evidenciam que pessoas com controle pressórico inadequado avaliaram negativamente os resultados referentes ao domínio $\mathrm{EF} 1(2,88 \pm 1,43 \mid \mathrm{p}=0,035)$ e ao domínio EF3 $(2,79 \pm 1,40 \mid \mathrm{p}=0,011)$ (tabela 2). 
Tabela 2. Distribuição da média do escore, desvio padrão e intervalo de confiança referentes aos atributos do enfoque familiar e orientação comunitária, da Atenção Primária à Saúde, por usuários com Hipertensão Arterial de acordo com o controle pressórico. Maringá, Paraná, Brasil, 2016

\begin{tabular}{|c|c|c|c|c|c|c|c|}
\hline \multirow[b]{3}{*}{ Indicadores } & \multirow{2}{*}{\multicolumn{2}{|c|}{ Total }} & \multicolumn{4}{|c|}{ Controle Pressórico } & \multirow[b]{3}{*}{$p$} \\
\hline & & & \multicolumn{2}{|c|}{ Adequado $(n=224)$} & \multicolumn{2}{|c|}{ Inadequado $(n=193)$} & \\
\hline & $\mathrm{M} \pm \mathrm{SD}$ & IC $95 \%$ & $\mathrm{M} \pm \mathrm{SD}$ & IC $95 \%$ & $\mathrm{M} \pm \mathrm{SD}$ & IC $95 \%$ & \\
\hline \multicolumn{8}{|c|}{ Enfoque na Família } \\
\hline EF1 & $3,04 \pm 1,45$ & $2,90-3,18$ & $3,18 \pm 1,46$ & $2,99-3,37$ & $2,88 \pm 1,43$ & $2,67-3,08$ & 0,035 \\
\hline EF2 & $3,78 \pm 1,40$ & $3,65-3,92$ & $3,90 \pm 1,40$ & $3,71-4,08$ & $3,64 \pm 1,38$ & $3,45-3,84$ & 0,065 \\
\hline EF3 & $2,98 \pm 1,45$ & $2,85-3,13$ & $3,16 \pm 1,47$ & $2,96-3,35$ & $2,79 \pm 1,40$ & $2,58-2,99$ & 0,011 \\
\hline \multicolumn{8}{|c|}{ Orientação Comunitária } \\
\hline $\mathrm{OC1}$ & $3,07 \pm 1,47$ & $2,93-3,22$ & $3,24 \pm 1,48$ & $3,05-3,44$ & $2,88 \pm 1,44$ & $2,67-3,08$ & 0,012 \\
\hline OC2 & $3,79 \pm 1,25$ & $3,67-3,91$ & $3,90 \pm 1,40$ & $3,73-4,06$ & $3,66 \pm 1,23$ & $3,48-3,84$ & 0,053 \\
\hline $\mathrm{OC3}$ & $3,72 \pm 1,31$ & $3,60-3,85$ & $3,86 \pm 1,30$ & $3,69-4,03$ & $3,56 \pm 1,30$ & $3,37-3,74$ & 0,017 \\
\hline
\end{tabular}

Fonte: Elaboração própria.

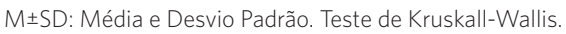

Quanto ao atributo orientação à comunidade, a maioria dos entrevistados avaliou como regular a questão relacionada com o incentivo dos profissionais de saúde sobre a importância de sua participação em eventos comunitários, como em igrejas, associações de bairro, eventos escolares, como apoio para o tratamento da doença (OC1) $(3,07 \pm 1,47)$, seguido do desenvolvimento de ações sobre HA em igrejas, associações de bairro e escolas (OC3) $(3,72 \pm 1,31)$. Observa-se que, na análise de variância, as pessoas que avaliaram negativamente $\mathrm{OC} 1$ $(2,88 \pm 1,44 \mid \mathrm{p}=0,012)$ e OC3 $(3,56 \pm 0,09 \mid \mathrm{p}$
$=0,017)$ são as classificadas com controle pressórico inadequado (tabela 2).

Para maior compreensão da variação das médias para os entrevistados com controle pressórico adequado e inadequado, são apresentadas as variações de médias dos grupos descritos por meio do diagrama de caixa. Em relação à posição dos dados, observa-se o segundo quartil dos domínios EF1, EF3 e OC1 na linha da nota três, sendo considerada regular na visão de pessoas com controle pressórico adequado. Os demais domínios (EF2, OC2 e OC3) mantêm o segundo quartil ou mediana na linha entre a nota três e quatro (figura 1). 
Figura 1. Diagrama de caixa das questões referentes ao enfoque familiar e orientação comunitária analisadas segundo o grupo de pessoas classificadas com controle pressórico adequado ( $n=224)$. Maringá, Paraná, Brasil, 2016

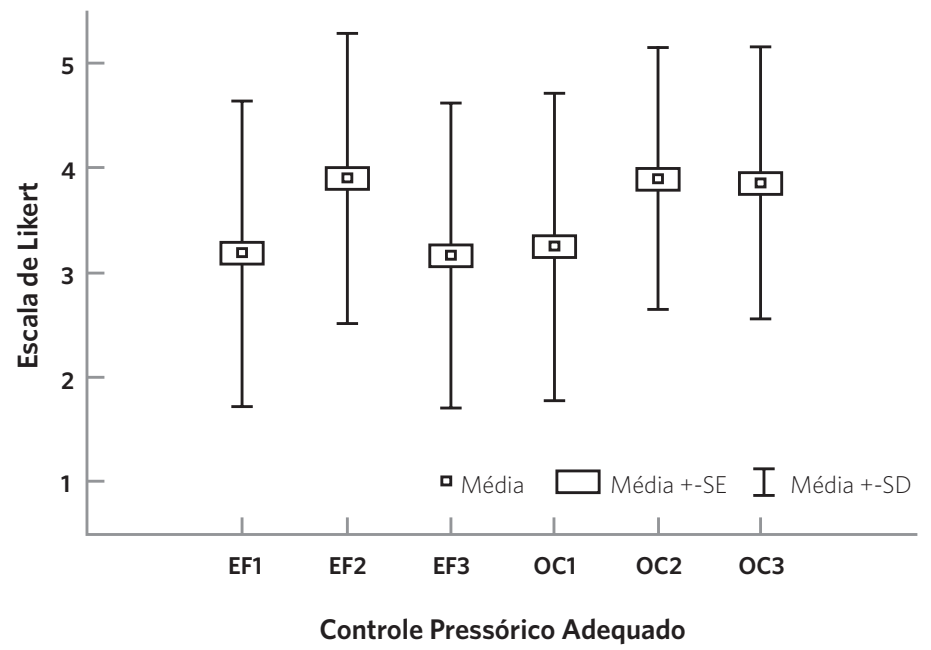

Fonte: Elaboração própria

No diagrama de caixa dos indivíduos classificados com controle pressórico inadequado, o quartil dos domínios EF1, EF3 e OC1 estão entre as notas dois e três, interpretados como insatisfatório; e os domínios EF2, OC2 e OC3 estão entre as notas três e quatro (figura 2). Reitera-se que, nos diagramas de caixa, não é observada a presença de outliers, não havendo valores discrepantes nos resultados.

Figura 2. Diagrama de caixa das questões referentes ao enfoque familiar e orientação comunitária analisadas segundo o grupo de pessoas classificadas com controle pressórico inadequado $(n=193)$. Maringá, Paraná, Brasil, 2016

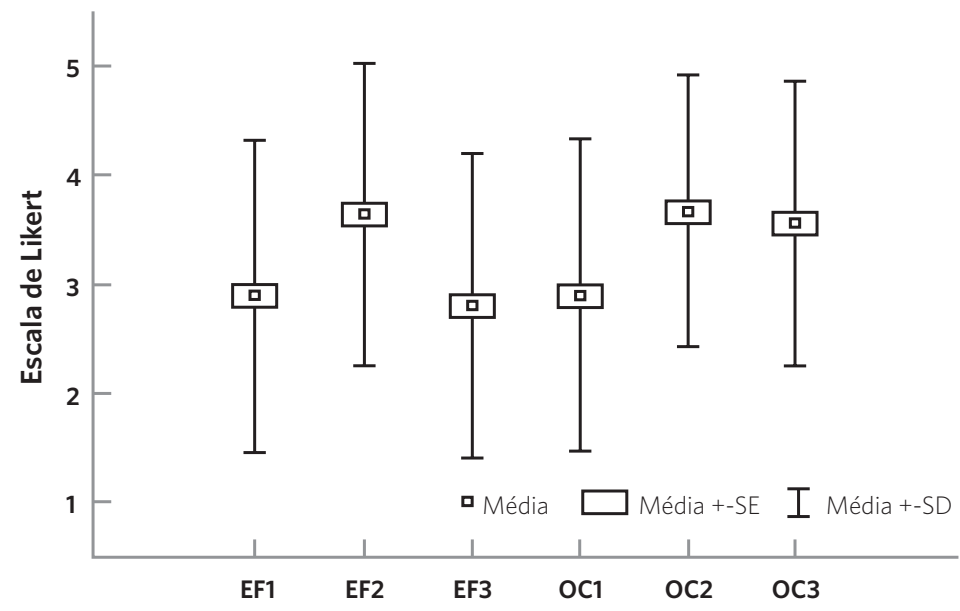

Controle Pressórico Inadequado 


\section{Discussão}

A maioria dos entrevistados era de idosos, do sexo feminino e da cor branca, o que não diferenciou entre o grupo de controle pressórico adequado e inadequado. Esses dados corroboram estudo desenvolvido no Rio Grande do Sul4, que avaliou a adesão ao tratamento entre seus entrevistados. Essas informações reforçam que a HA é prevalente na maioria das pessoas com idade mais avançada devido ao aumento da expectativa de vida e das questões fisiológicas que propiciam o surgimento de doenças crônicas ${ }^{\mathbf{1}, \mathbf{2}, \mathbf{4}}$.

O fato de a população feminina ser mais prevalente nesta pesquisa reforça os resultados de dois estudos de base populacional1,3, os quais consideram que a mulher tem melhor percepção sobre o estado de saúde e sobre o próprio corpo com mais precisão e procura serviços de saúde com mais prevalência. Em relação a questões étnico-raciais, esta pesquisa encontrou divergência em estudo ${ }^{1}$ de base populacional, em que houve a prevalência de pessoas que se autodeclaravam negras. Compreende-se que, apesar das disparidades entre os dois estudos, reforça-se o manejo das ações de saúde, independentemente de perfil étnico, reiterando a necessidade de considerar que complicações da HA são comumente mais frequentes em população negra ${ }^{2}$.

Entre os entrevistados, grande parte tinha até o ensino fundamental, era casada, pertencia à classe econômica extrato $\mathrm{C} 2$ e era aposentada e/ou pensionista. Esses dados corroboram estudo 5 realizado na Índia, em que a população menos escolarizada e com menor renda está mais propícia a apresentar inadequação ao controle pressórico e a compreender as orientações que são fornecidas pelos profissionais de saúde, havendo necessidade de identificação e ações de educação em saúde mais assertivas a essa parcela da população ${ }^{\mathbf{5}}$.

O instrumento utilizado na coleta de dados deste estudo, por ter um alcance abrangente, propiciou a apresentação de questões relacionadas com o enfoque familiar como estratégia de apoio social para o tratamento da HA. Nesse ponto, os entrevistados avaliaram como incipiente o interesse dos profissionais de saúde em conhecer a família e incorporá-la ao tratamento.

Resultados semelhantes foram encontrados em estudo realizado em Manaus ${ }^{10}$, no qual os entrevistados relataram pouco interesse dos profissionais de saúde para o conhecimento e envolvimento da família no tratamento, e que a abordagem permaneceu no indivíduo, como foco da atenção. Essas ações realizadas pelos profissionais, em que a família é pouco incorporada ao manejo das práticas assistenciais, acabam desconsiderando a característica intervencionista que o arrojo familiar possui ${ }^{18}$.

Ainda no enfoque familiar, a maioria dos entrevistados avaliou como regular a orientação dos profissionais de saúde sobre a importância do envolvimento da família no tratamento. Esses resultados se assemelham a estudo 9 realizado na capital da Paraíba, em que a maioria dos entrevistados dos cinco distritos investigados apresentou satisfação regular com o desempenho citado.

A participação da família no tratamento de doenças crônicas, especialmente a HA, temática deste estudo, pode ser o facilitador da aderência à terapêutica de escolha por se constituir no apoio social do membro adoecido e por incentivar a adoção de práticas de autocuidado. Barreto e Marcon ${ }^{18}$, ao avaliar a participação da família de pessoas com HA, no tratamento da morbidade, constataram que a família contribui para as questões culturais e que podem ser integradas às orientações realizadas pelos profissionais de saúde quanto ao entendimento dessas ações e ao incentivo em realizá-las.

A análise de dados revelou que houve diferenças significativas na questão do envolvimento da família no tratamento, em que pessoas com controle pressórico inadequado são mais suscetíveis a avaliar insatisfatoriamente o desempenho dos profissionais de saúde nesse indicador proferido. Em estudo ${ }^{\mathbf{1 8}}$ realizado no interior do estado do Paraná, 
as dificuldades da família em envolver-se nas práticas de autocuidado e de manejo das orientações de saúde ao membro adoecido prejudicavam a aderência ao regime terapêutico de escolha, acarretando inadequação no controle pressórico.

Segundo a Política Nacional da Atenção Básica (PNAB) ${ }^{6}$, a família deve ser integrada nas intervenções realizadas para o controle de qualquer doença, considerando que é no arranjo familiar que se concentram as práticas de saúde baseadas no aparato cultural, que podem estar fragmentadas ao modelo biomédico, da cura centrada na doença e que deve ser corrigida. Nesse contexto, Rêgo e Radovanovic ${ }^{\mathbf{8}}$ reiteram a importância da escuta terapêutica e das orientações para o tratamento, havendo criação de vínculo entre profissional e usuário, resultando em uma melhor aderência ao tratamento e adequação ao controle pressórico.

Com relação à adesão/vínculo, a família deve ser o foco da atenção e deve abordada como constituinte do processo de construção do cuidado, com abordagens que favoreçam o tratamento. Em estudo conduzido por Brito e colaboradores, realizado com trabalhadores pertencentes da ESF e do Núcleo de Apoio à Saúde da Família (Nasf), para abordagem ao familiar adoecido, é necessário que os profissionais de saúde verifiquem quais são suas necessidades pessoais, avaliem as questões relacionadas com o ambiente em que este está inserido, a disposição de materiais e as condições em que o cuidado é realizado, o que poderá facilitar a compreensão do processo de cuidado, tanto dos profissionais quanto dos membros familiares e do indivíduo adoecido ${ }^{12}$.

Quanto à orientação comunitária, a maioria dos entrevistados avaliou de forma regular o domínio referente ao incentivo dos profissionais de saúde na sua participação em ações sociais, como associações de bairros, igrejas e escolas. Esse resultado corrobora estudo9 realizado em João Pessoa, no estado da Paraíba, sobre sua incumbência nos processos que envolvem a participação popular.
Reitera-se que esse domínio estava relacionado estatisticamente com o grupo de pessoas com controle pressórico inadequado, sedo avaliado insatisfatoriamente.

A participação social é uma concepção organizacional do Sistema Único de Saúde (SUS) que favorece a construção de usuários proativos, com capacidade de analisar seu contexto e desenvolver estratégias de enfrentamento e cuidado da sua doença crônica, no caso, HA. Além disso, propicia a construção coletiva de práticas de autocuidado a partir da troca de experiências, amparadas na ajuda e na solidariedade, de acordo com as orientações clínicas adequadas ${ }^{19}$.

Concomitantemente a isso, a participação social possibilita a produção da autonomia no cuidado da própria saúde, na construção da cidadania e na busca por direito à saúde de acordo com suas necessidades. No entanto, alguns profissionais de saúde ainda geram práticas que podem fragilizar a construção da independência dos usuários dos serviços de saúde quando não apresentam e incentivam a prática e modos de tecer a busca por cuidado"1.

Nesse aspecto, compreende-se que a participação das pessoas em ações sociais na comunidade permeia a construção de consciência a respeito da saúde, reflexões relacionadas com a temática, ocasionando movimentos em prol de mudanças nas políticas de saúde coletiva que podem potencializar as aplicações de recursos públicos para a necessidade de saúde das pessoas e da comunidade envolvida ${ }^{19}$.

Os resultados deste estudo ainda apontam que a maioria dos entrevistados avaliou insatisfatoriamente a realização de ações de educação em saúde em associações de bairros, igrejas e escolas, estando significativamente atreladas ao grupo de pessoas com controle pressórico inadequado. Esses resultados assemelham-se aos dados da avaliação externa do Programa Nacional de Melhoria do Acesso e da Qualidade da Atenção Básica (PMAQ-AB) ${ }^{20}$.

A necessidade de educação em saúde é atenuante no País e palco de políticas públicas, principalmente pela expansão da EFS nos 
últimos anos, sendo crucial para o conhecimento e construção do cuidado do usuário e da comunidade sobre questões relacionadas com a saúde, e doenças crônicas ${ }^{\mathbf{2 0} 21}$. Em estudo realizado com 816 equipes da ESF no Rio Grande do Sul que aderiram ao PMAQ-AB, foram efetivadas orientações e educação em saúde visando hábitos de vida saudáveis, práticas de atividades físicas e alimentares, mas com foco nas pessoas com doenças crônicas já instaladas e com hábitos de vida inadequado, e não focadas na promoção da saúde ${ }^{21}$.

Este estudo não possui o foco de determinar que tipo de atividade educativa é realizada, mas se há incentivo à prática de educação em saúde para a comunidade, limitado a informações contidas no instrumento de coleta de dados. Dessa forma, tendo o contexto que são realizadas as atividades pertinentes aos grupos de Sishiperdia, a oferta ainda é centrada nas doenças e não explora questões relativas à comunidade; e, sim, a uma população específica, havendo necessidade de ações para alterar esta realidade.

É necessário que novas abordagens a determinantes sociais sejam criadas e implementadas, com organização do processo de trabalho, rompendo paradigmas de práticas individualizadas. Dessa forma, pode-se contribuir para a construção do compromisso em torno da educação em saúde, com estímulo da participação popular e ampliação do cuidado e controle da própria saúde $20,21$.

Este estudo aponta que as atitudes dos profissionais de saúde necessitam desempenhar a integralidade do cuidado, com ruptura das práticas voltadas ao modelo biomédico, de atenção e cuidado fragmentado. Os esforços para estimular novas práticas em saúde no contexto familiar e comunitário devem ser assertivos e que atendam à necessidade da população, comprometidos com a qualidade de vida dessas pessoas ${ }^{12,22}$.

As ações desenvolvidas pelos profissionais de saúde devem ser fundamentadas na intersetorialidade, alcançando áreas além das UBS, com predomínio do contexto social, econômico, político, cultural e na conjuntura coletiva da comunidade; garantindo, com isso, a promoção da autonomia e a capacidade de autocuidado, com ações resolutivas em saúde 22 .

Este estudo limita-se ao aspecto geográfico por ter sido realizado em apenas um município, o que impede sua generalização para os demais estados do País. No entanto, os achados podem contribuir para o fortalecimento das ações em saúde, abarcando a integralidade do sujeito dentro do seu contexto familiar e encontrando meios dinâmicos que informem a comunidade sobre seu papel reivindicatório, com incentivo a mudanças na gestão pública dos serviços ofertados pelo município, reforçando as práticas de educação em saúde. Além disso, pode subsidiar novas metodologias de ensino, construindo novos modelos operacionais com as fragilidades apontadas nesta pesquisa.

\section{Conclusões}

O estudo permitiu identificar que a maioria dos entrevistados avaliou insatisfatoriamente os domínios relacionados com o conhecimento dos profissionais de saúde sobre os integrantes e o incentivo da família no tratamento da HA. No contexto comunitário, os participantes avaliaram insatisfatoriamente o incentivo dos profissionais de saúde sobre sua participação em ações sociais desenvolvidas por igrejas, associações de bairros e escolas. Ademais, avaliaram como regular o desenvolvimento de ações de educação em saúde nessas mesmas instituições.

O estudo aponta necessidades de preparar melhor os futuros profissionais para desenvolverem uma assistência integral e resolutiva, de forma equânime, com conhecimento da realidade de cada indivíduo e da comunidade envolvida. Destaca-se a importância de trabalhar com os profissionais de saúde, identificando suas necessidades, incentivando-os a organizar o trabalho entre as equipes com discussões e estudo de caso, 
principalmente dos usuários com HA, para que reduzam as iniquidades da assistência, promovendo melhores condições de trabalho e melhor qualidade assistencial à população aderente aos serviços da APS.

Reitera-se a importância de os profissionais de saúde trabalharem com vista à integralidade da assistência e, em equipe, executarem o planejamento de práticas educativas inovadoras, intentando a promoção e a educação em saúde, buscando empoderar a comunidade para gerir o autocuidado necessário à condição crônica.

\section{Referências}

1. Zangirolani LTO, Assumpção D, Medeiros MAT, et al. Self-reported hypertension in adults residing in Campinas, Brazil: prevalence, associated factors and control practices in a population-based study. Ciênc. Saúde Colet. [internet]. 2018 [acesso em 2018 set 12]; 23:1221-1232. Disponível em: http://dx.doi. org/10.1590/1413-81232018234.16442016.

2. Perini W, Agyemang C, Snijder MB, et al. Ethnic disparities in treatment rates for hypertension and dyslipidemia: an analysis by different treatment indications the Healthy Life in an Urban Setting study. J. hypertens. [internet]. 2018 [acesso em 2018 set 12]; 36(7):1540-1547. Disponível em: http://dx.doi. org/10.1097/HJH.0000000000001716.

\section{Colaboradores}

Santos FGT (0000-0001-7082-6949)*, Rêgo AS (0000-0002-0988-5728)* e Radovanovic CAT (0000-0001-9825-3062)* participaram da concepção, delineamento, análise e interpretação dos dados; redação revisão crítica do artigo e aprovação da versão submetida. Salci MA(0000-0002-6386-1962)* e Mezzavila VAM (0000-0001-8163-7908)* participaram da análise e interpretação dos dados, redação e revisão crítica do artigo e aprovação da versão final. e Agravos não Transmissíveis e Promoção da Saúde. Vigitel Brasil 2017: vigilância de fatores de risco e proteção para doenças crônicas por inquérito telefônico: estimativas sobre frequência e distribuição sociodemográfica de fatores de risco e proteção para doenças crônicas nas capitais dos 26 estados brasileiros e no Distrito Federal em 2017. Brasília, DF: Ministério da Saúde; 2018.

4. Gewehr DM, Bandeira VAC, Gelatti GT, et al. Adesão ao tratamento farmacológico da hipertensão arterial na Atenção Primária à Saúde. Saúde debate [internet]. 2018 [acesso em 2018 set 12]; 42:179-90. Disponível em: http://dx.doi.org/10.1590/0103-1104201811614.

5. Balasubramanian A, Nair SS, Rakesh P, et al. Adherence to treatment among hypertensives of rural Kerala, India. J Family Med Prim Care [internet]. 2018 
[acesso em 2018 set 13]; 7(1):64-69. Disponível em: http://dx.doi.org/10.4103/jfmpc.jfmpc_423_16.

6. Brasil. Ministério da Saúde. Política Nacional de Atenção Básica. Brasília, DF: Ministério da Saúde; 2012.

7. Starfield B. Atenção primária: equilíbrio entre necessidades de saúde, serviços e tecnologia. Brasília: Unesco, Ministério da Saúde; 2002.

8. Rêgo AS, Radovanovic CAT. Adherence of hypertension patients in the Brazil's Family Health Strategy. Rev. bras. enferm. [internet]. 2018 [acesso em 2018 set 14]; 71(3):1030-1037. Disponível em: http://dx.doi. org/10.1590/0034-7167-2017-0297.

9. Araújo JSS, Silva CS, Paes NA, et al. Satisfação de mulheres hipertensas na atenção primária com relação aos atributos essenciais família e comunidade. Saúde debate [internet]. 2015 [acesso em 2018 set 14]; 39:411422. Disponível em: http://dx.doi.org/10.1590/0103110420151050002009 .

10. Silva NCd, Giovanella L, Mainbourg EMT. The family in the practices of Family Health teams. Rev. bras. Enferm. [internet]. 2014 [acesso em 2018 set 16]; 67(2):274-81. Disponível em: http://dx.doi. org/10.5935/0034-7167.20140037.

11. Mendes R, Fernandez JCA, Sacardo DP. Promoção da saúde e participação: abordagens e indagações. Saúde debate [internet]. 2016 [acesso em 2018 set 18]; 40:190203. Disponível em: http://dx.doi.org/10.1590/01031104-20161080016.

12. Brito GEG, Mendes ACG, Santos Neto PM. Purpose of work in the Family Health Strategy. Interface (Botucatu, Online) [internet]. 2018 [acesso em 2018 set 14]; 22(64):77-86. Disponível em: http://dx.doi. org/10.1590/1807-57622016.0672.

13. Conselho Nacional de Saúde. Resolução número 466, de 12 de dezembro de 2012. Diário Oficial da União. 12 Dez 2012.

14. Instituto Paranaense de Desenvolvimento Econômico e Social. Caderno Estatístico Município de Maringá [internet]. Curitiba: Ipardes; 2016 [acesso em 2018 set 16]. Disponível em: http://www.ipardes.gov.br/cadernos/MontaCadPdfl.php?Municipio=87000\&btOk=ok.

15. Associação Brasileira de Empresas de Pesquisa. Adoção do CCEB 2008: critério de Classificação Econômica Brasil. São Paulo: Abep; 2015.

16. Paes NA, Silva CS, Figueiredo TMRM, et al. Satisfação dos usuários hipertensos com os serviços da rede de atenção primária no Brasil: um estudo de validação. Rev. panam. salud pública [internet]. 2014 [acesso em 2018 set 14]; 36(2):87-93. Disponível: http:// www.scielosp.org/pdf/rpsp/v36n2/03.pdf.

17. Malachias MVB, Souza WKSB, Plavnik FL, et al. $7^{\mathrm{a}}$ Diretriz Brasileira de Hipertensão Arterial. Arq. bras. cardiol. [internet]. 2016 [acesso em 2018 set 16]; 107(3):1-104. Disponível em: http://www.scielo.br/ pdf/abc/v107n3s3/0066-782X-abc-107-03-s3-0067. pdf.

18. Barreto MS, Marcon SS. Patient perspectives on family participation in the treatment of hypertension. Texto \& contexto enferm. [internet]. 2014 [acesso em 2018 set 17]; 23(1):38-46. Disponível em: http:// dx.doi.org/10.1590/S0104-07072014000100005.

19. Lima FA, Galimbertti PA. Sentidos da participação social na saúde para lideranças comunitárias e profissionais da Estratégia Saúde da Família do território de Vila União, em Sobral-CE. Physis (Rio J.) [internet]. 2016 [acesso em 2018 set 17]; 26(1):157175. Disponível em: http://dx.doi.org/10.1590/S010373312016000100010 .

20. Medina M, Aquino R, Vilasbôas ALQ, et al. Health Promotion and Chronic Disease Prevention: What Are Family Health Teams Doing? Saúde debate [internet]. 2014 [acesso em 2018 set 15]; 38(esp):6982. Disponível em: http://www.scielo.br/pdf/sdeb/ v38nspe/0103-1104-sdeb-38-spe-0069.pdf.

21. Kessler M, Thumé E, Duro SMS, et al. Ações educativas e de promoção da saúde em equipes do Programa Nacional de Melhoria do Acesso e da Qualidade da Atenção Básica, Rio Grande do Sul, Brasil. Epide- 
miol. serv. saúde [internet]. 2018 [acesso em 2018 set 16]; 27(2):1-12. Disponível em: https://doi.org/10.5123/ S1679-49742018000200019.

22. Viegas SMF, Penna CMM. The dimensions of the comprehensiveness on healthcare within the routine of the Family Health Strategy in the Jequitinhonha Valley, Minas Gerais, Brazil. Interface (Botucatu, Online) [internet]. 2015 [acesso em 2018 set 17]; 19(55):1089100. Disponível em: http://dx.doi.org/10.1590/180757622014.0275 .

Recebido em 30/09/2018

Aprovado em 02/04/2019

Conflito de interesses: inexistente

Suporte financeiro: Coordenação de Aperfeiçoamento de Pessoal de Nível Superior (Capes) 